\title{
A Case of Male Anorgasmia with Cerebral Infarction
}

\author{
Masato Shirai", Yoshio Kawachi, Shigeto Yanada, Hajime Watanabe, Youichiro Toyonaga, \\ Sousuke Sugimura, Toru Sakurai, Makoto Fujime \\ Department of Urology, Juntendo University Urayasu Hospital, Chiba, Japan \\ Email: "masatos@juntendo-urayasu.jp
}

Received May 28, 2012; revised June 25, 2012; accepted July 5, 2012

\begin{abstract}
Objective: The deficit of male orgasm is an unusual clinical condition. This is a case report on secondary male anorgasmia with cerebral infarction. Material and Method: The patient was a 55-year-old man who had suffered from male anorgasmia, acquired type. The patient had a normal erectile response, but ejaculated without any sensation of orgasm. The patient underwent an evaluation by a psychiatrist and an urologist trained in sexual medicine. Result: The results of physical, psychological, and laboratory examination were normal. Brain MRI revealed cerebral infarction at bilateral parietal lobes and frontal lobes. Conclusion: There is a possibility that these brain regions are related to male orgasmic dysfunction.
\end{abstract}

Keywords: Male Anorgasmia; Orgasmic Dysfunction; Cerebral Infarction; Brain Imaging

\section{Introduction}

The deficit of male orgasm is an unusual clinical condition with unknown etiology. For difficulties in reaching orgasm, lifetime prevalence estimates a range of up to $34 \%$ in women [1]. But male anorgasmia is extremely rare, especially secondary anorgasmia [2]. In the present report, we report a case of male anorgasmia and the possibility of an effect of brain regions is discussed.

\section{Case Report}

A 55-year-old man visited our hospital in 2006 complaining of a history of loss of orgasmic sensation since 1991. The patient underwent an evaluation by a psychiatrist and an urologist trained in sexual medicine. He had had no family history of appreciable disease. He had had the past history of Vestibular neuronitis (1990), and Hypertension (1996-2006). He had been taking Betaxolol (beta-blocker) $20 \mathrm{mg} /$ day since 1996 . His sexual history was normal. He did not have a sexual partner at that time. His libido, erection, and arousal were normal. But he ejaculated without any sensation of orgasm. His penis, testicles, epididymides, prostate, sensory examination, bulbocavernosus reflex, and anal sphincter reflex were normal. The result of International Index of Erectile Function-5 was 24 points (normal range is between 22 and 25 points). The blood test including hormonal test was all normal. The psychological state that was evaluated by a psychiatrist was normal. The brain MRI re-

${ }^{*}$ Corresponding author. vealed cerebral infarction at bilateral parietal lobes and frontal lobes (Figure 1). We gave Amantadine Hydrochloride $100 \mathrm{mg}$ /day to this patient from January 2008 to July 2008 [3]. But, there was no effect. We proposed to him to change another drug include Oxytocin nasal spray [4]. But, he refused to take any other drugs. He had received only counseling. His ability of erection and ejaculation remained the same. But, he ejaculated without any orgasmic sensation.

\section{Discussion}

Definition of anorgasmia is absence of orgasm experience, independent of whether or not any or all of the physiologic concomitants of ejaculation [2]. The possible causes of anorgasmia are, psychogenic, congenital, anatomic, neurogenic, infective, endocrine, and medication [2]. We report a case that had normal sexual responses without orgasmic sensation. His libido, erection, arousal, reflex, and penile sensory were normal. The results of the blood test were also normal. A psychiatrist denied any psychological cause. To the best of our knowledge, we could not find out the relationship between anorgasmia and Betaxolol, because the onset of anorgasmia was prior to starting medication, and his symptom did not change after taking medication. Betaxolol is not thought to be associated with anorgasmia. Only the brain MRI revealed an abnormal condition. To the best of our knowledge, we could not find out any other case reports of male anorgasmia with diagnostic imaging such as brain MRI. In animal studies, orgasm is 

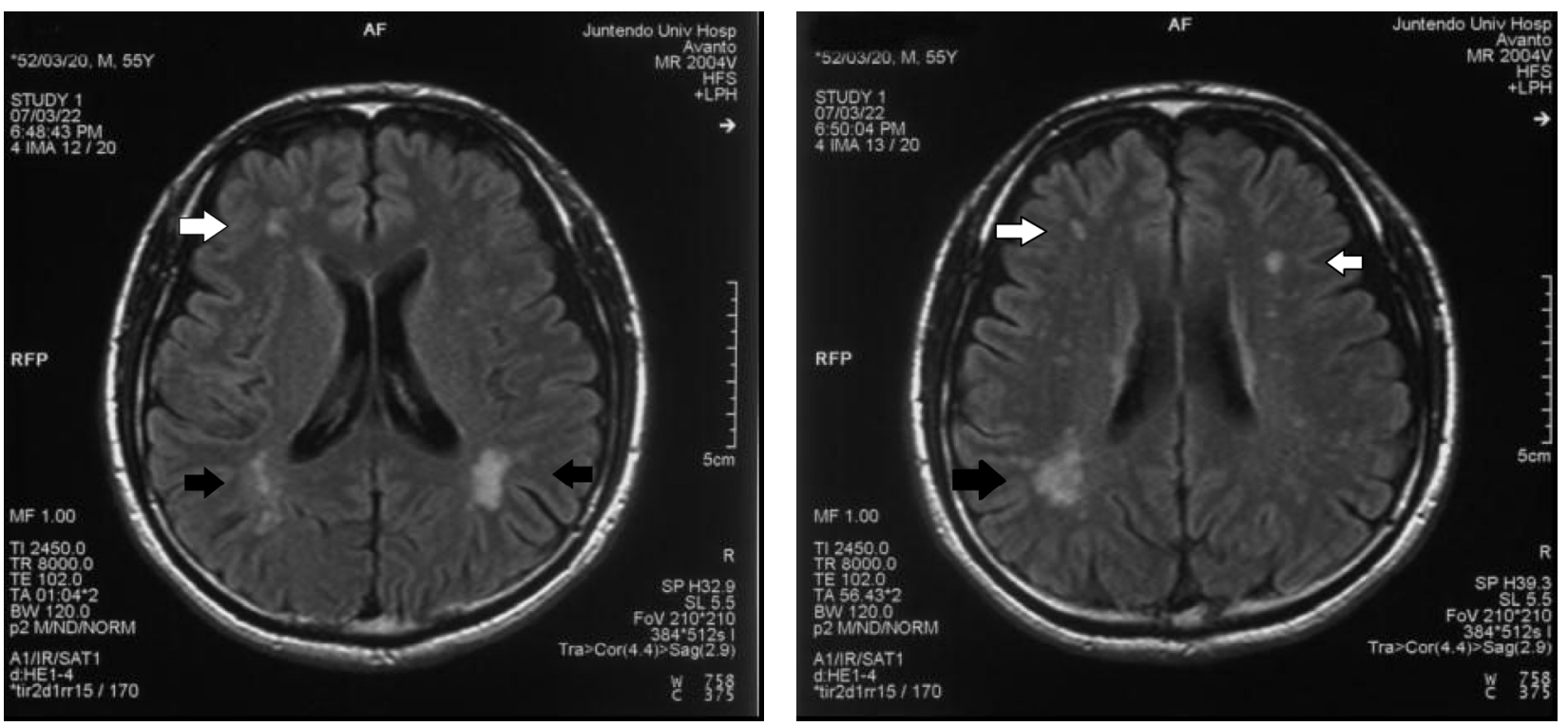

Figure 1. Brain MRI revealed cerebral infarction at bilateral parietal lobes (black arrows) and frontal lobes (white arrows).

associated with the medial preoptic area and paraventricular nucleus [5]. But in humans, we do not have enough results about orgasm with diagnostic imaging. Georgiadis et al. reported on brain activation during healthy male ejaculation using positron emission tomography [6]. They demonstrated ejaculation-related activations in the dentate nucleus, anterior vermis, pons, and ventrolateral thalamus, and ejaculation-related deactivations in the prefrontal cortex. In general, the regions at the bilateral parietal lobes and frontal lobes that are revealed by brain MRI are not thought to be associated with subjective symptoms [7]. The regions are detected by coincidence with MRI or CT. The dentate nucleus, anterior vermis, pons, ventrolateral thalamus, and prefrontal cortex were normal in this patient. But because some brain functions are still being discovered, the effect of cerebral infarction cannot be denied relating to anorgasmia in this patient. We still need a definitive method that objectively measures orgasm. Treatment of anorgasmia has met with limited success. Psychosexual Therapy is recommended for anorgasmia [2]. In contrast, pharmacological agents, including Amantadine, Yohimbin, Apomorphine, and Oxytocin increase the ejaculation in men with orgasmic disorder [2]. In this patient, Amantadine Hydrochloride was no effect. We need a definitive therapy for anorgasmia by a series of case reports brought together.

\section{REFERENCES}

[1] J. L. Shifren, B. U. Monz, P. A. Russo, et al., "Sexual
Problems and Distress in United States Women: Prevalence and Correlates," Obstetrics and Gynecology, Vol. 112, No. 5, 2008, pp. 970-978. doi:10.1097/AOG.0b013e3181898cdb

[2] D. Rowland, C. G. McMahon, C. Abdo, et al., "Disorders of Orgasm and Ejaculation in Men," Journal of Sexual Medicine, Vol. 7, No. 4, 2010, pp. 1668-1686. doi:10.1111/j.1743-6109.2010.01782.x

[3] A. A. Keller, R. Hamer and R. C. Rosen, "Serotonin Reuptake Inhibitor-Induced Sexual Dysfunction and Its Treatment: A Large-Scale Retrospective Study of 596 Psychiatric Outpatients," Journal of Sex \& Marital Therapy, Vol. 23, No. 3, 1997, pp. 165-175. doi: $10.1080 / 00926239708403922$

[4] W. W. IsHak, D. S. Berman and A. Peters, "Male Anorgasmia Treated with Oxytocin," Journal of Sexual Medicine, Vol. 5, No. 4, 2008, pp. 1022-1024. doi:10.1111/j.1743-6109.2007.00691.x

[5] J. P. Heaton and M. A. Adams, "Update on Central Function Relevant to Sex: Remodeling the Basis of Drug Treatments for Sex and the Brain," International Journal of Impotence Research, Vol. 15, Suppl. 5, 2003, pp. 25-32. doi:10.1038/sj.ijir.3901069

[6] J. R. Georgiadis, A. A. Reinders, F. H. van der Graaf, et al., "Brain Activation during Human Male Ejaculation Revisited," Neuroreport, Vol. 18, 2007, pp. 553-557. doi:10.1097/WNR.0b013e3280b10bfe

[7] M. W. Vernooij, M. A. Ikram, H. L. Tanghe, et al., "Incidental Findings on Brain MRI in the General Population," New England Journal of Medicine, Vol. 357, 2007, pp. 1821-1828. doi:10.1056/NEJMoa070972 